\title{
Transition Probabilities for Trichoplusia ni (Lepidoptera: Noctuidae) Larvae on Cabbage as a Function of Microclimate
}

\author{
CASEY W. HOY, ${ }^{1}$ C. E. MCCULLOCH ${ }^{2}$ C. A. SHOEMAKER, ${ }^{3}$ \\ AND A. M. SHELTON \\ Department of Entomology, Cornell University, \\ New York State Agricultural Experiment Station, \\ Geneva, New York 14456
}

\begin{abstract}
Environ. Entomol. 18(2): 187-194 (1989)
ABSTRACT To identify factors affecting the spatial dynamics of Trichoplusia ni (Hübner) on cabbage, the movements of individual larvae were monitored. Larvae were marked with ${ }^{32} \mathrm{P}$ and released individually, one per plant, on cabbage plants in research plots. Their movements were monitored by recording their position on the plant daily, locating them with a Geiger counter. In the same plots, hourly measurements of temperature and relative humidity were recorded in three different parts of the crop canopy. Transition probabilities for the larvae from and to each of five vertical plant strata were modeled as definite integrals of the Beta probability density function (pdf). The shape parameters for these Beta pdf's were modeled as a function of microclimate, and the necessary parameters to do so were estimated by the method of maximum likelihood. This model predicted the data well and was more efficient than logistic regression. The model predicts that under high temperatures and low vapor pressure deficit (vpd), larvae move down to the shaded, cooler, lower parts of the plant; whereas under low temperatures and high vpd, they moved upward toward the economically important wrapper leaves and cabbage heads.
\end{abstract}

KEY WORDS Insecta, spatial dynamics, modeling, dispersal

MANY INSECT PESTS damage different parts of their host plants. In some cases all the damaged parts are economically important, but in most crops, only a certain part of the plant will be harvested, and damage on other parts does not directly affect marketability of the crop. Predicting when pests will damage the marketable parts is essential for economical control, for without such predictions, the grower must assume a constant risk of direct damage. Predicting this damage, however, may require that intraplant movements of the insect pest be forecast, because the location of damage on the plant is a function of the location of the pests. Movements of insects on their host plant could be influenced by factors in the host plant itself or in the surrounding environment, which makes it a difficult process to predict.

An example of a pest that sometimes damages the marketable parts of its host plant and sometimes does not is the cabbage looper, Trichoplusia ni (Hübner), on cabbage. Reports in the literature (Alwood 1886, Sirrine 1894, Hayslip et al. 1953, Reid \& Cuthbert 1957, Sutherland 1965, Greene

'To whom reprint requests should be addressed. Current address: Department of Entomology, Ohio Agricultural Research and Development Center, Wooster, Ohio 44691.

'Biometrics Unit, Cornell University, Ithaca, N.Y. 14853

${ }^{3}$ Department of Civil and Environmental Engineering, Cornell University, Ithaca, N.Y. 14853.
1968) suggest that late instars of $T . n i$ move from the frame leaves, where the majority of the eggs are laid, to the head and wrapper leaves (adjacent to the head) and feed there. Only the head and four surrounding wrapper leaves are harvested, and damage on these leaves directly affects marketability (Anonymous 1945). However, Hoy \& Shelton (1987) showed that late instars of T. ni do not prefer to feed on these leaves compared with the frame leaves in a laboratory choice test. Furthermore, the vast majority of $T . n i$ larvae are sometimes found on the lowest leaves on the plant (C.W.H., unpublished data).

Microclimate could affect the distribution of $T$. $n i$ on cabbage plants in the field. Hoy \& Shelton found that, in the laboratory, late instars would move away from a source of radiant heat (C.W.H. \& A.M.S., unpublished data). This response was affected by body size and ambient humidity; lower humidity and larger larval body size resulted in moving farther from the source of heat. Casey (1976) found that diurnal movements of two species of desert caterpillars on their host plants resulted in the maintenance of a temperature balance, and other insects have been shown to exhibit behaviors that result in a temperature balance (May 1979) or water balance (Cloudsley-Thompson 1962, 1975).

We hypothesize that microclimate in the cabbage crop canopy affects the movement of late 
instars of $T . n i$ on the plant. If true, the effect of microclimate on movement patterns of larvae within the crop canopy could determine the potential for damage to the economically important parts of the plant. The purpose of this paper is to describe how we tested this hypothesis (by testing the relationship of transition probabilities for larvae on the plant to microclimate) and how the same model used for the test can be used to predict the frequency of insect (or other biotic agent) movement as a function of environmental or other conditions.

\section{Materials and Methods}

Movements of individual $T$. ni larvae on cabbage plants in research plots were recorded by censusing their position on the plants daily. Larvae under observation were labeled with ${ }^{32} \mathrm{P}$ by the following procedure. Second or third instars were fed disks ( $2 \mathrm{~mm}$ diameter) of broccoli leaf placed on a $1-\mu l$ droplet of aqueous solution containing about $0.25 \mu \mathrm{Ci}$ of ${ }^{32} \mathrm{P}$ as orthophosphate in $\mathrm{HCl}-$ free solution. The larvae were held in plastic well plates for $24 \mathrm{~h}$ or until the leaf disks had been consumed, then were fed additional broccoli leaf and held for an additional $48 \mathrm{~h}$ before being released in research plots. Approximately $50 \%$ of the ${ }^{32} \mathrm{P}$ consumed was incorporated into the body of each larva, enough to label the larvae for the duration of the experiment.

One larva was released on every other plant within a row, and equal numbers were systematically released on each of five vertical strata and in each cardinal direction. The five vertical strata were defined as: (1) head, (2) wrapper leaves (the four loose leaves next to the head), (3) upper frame leaves (the four leaves below the wrapper leaves), (4) midframe leaves (the four to six leaves below the upper frame leaves), and (5) lower frame leaves (all leaves below the midframe leaves). The release strategy was to place a labeled larva on the north side of the cabbage head on the second plant in a row, another on a wrapper leaf on the east side of the fourth plant in the row, another on an upper frame leaf on the south side of the sixth plant in the row, etc.

Monitoring movements throughout a 24-h period indicated that fewest movements occurred during late afternoon and evening and that most larvae were feeding during this time. The daily census of larval positions on the plant was done during this time by systematically searching the plant with a Geiger-Müller tube to detect the location of the labeled larvae. When the location of the larva was identified and checked visually, exercising care not to disturb the larva, its position on the plant was recorded. Although movements between plants occurred, they were not of interest for the present study. If an interplant movement was accompanied by a change in stratum, however, this was recorded as an interstratum movement.
The data thus collected were the numbers of larvae not moving and moving from each stratum to each of the other strata each day.

Larvae that pupated or died were returned to the laboratory for disposal, and these were replaced with freshly labeled larvae periodically. Between 10 and 96 larvae were monitored daily from 13 August until 23 October 1986, with three interruptions of less than $5 \mathrm{~d}$ each. Observations taken within $2 \mathrm{~d}$ of pupation or of death were not used in the analysis. The cabbage varieties used were cv. King Cole for August and early September monitoring and $\mathrm{cv}$. Bartolo for mid-September and later. Plant spacings were $0.9 \mathrm{~m}$ between the rows and $0.46 \mathrm{~m}$ between plants within rows. Plots were maintained according to commercial practice and sprayed with methomyl (E. I. du Pont de Nemours \& Company, Wilmington, Del.) (in the early growth stages only) to prevent defoliation by other Lepidoptera. Monitoring was conducted when plants were in the late head fill growth stage (stage 8 , Andaloro et al. 1983).

While these movements were being monitored, temperature and relative humidity were being monitored in three different parts of the crop canopy and recorded. Thermistor temperature probes (Campbell Scientific, Inc., Logan, Utah) and metallic capacitive relative humidity probes (Thunder Scientific Corp., Albuquerque, N. Mex.) were connected to a weather data-logger (Campbell Scientific, Inc.), which recorded average hourly temperature and a sample of relative humidity at the end of each hour. Temperature and relative humidity probes were placed at the base of the head between the lower surface of the outermost head leaf and the upper surface of the adjacent wrapper leaf, underneath the upper frame leaves, and underneath the lowest frame leaves. These placements were designed to measure air temperature and humidity just above the leaf surface. Vapor pressure deficit ( $v p d)$ in these strata was estimated from relative humidity and temperature by

$$
\begin{gathered}
\operatorname{vpd}=\mathrm{e}_{s}-\mathrm{e}_{a} \\
\text { where } \mathrm{e}_{s}=0.61078 \exp \left(\frac{17.269 T}{T+237.30}\right), \mathrm{e}_{a}=\mathrm{e}_{s} \times
\end{gathered}
$$

$\frac{R H}{100}, T$ is temperature in ${ }^{\circ} \mathrm{C}, R H$ is the relative humidity, $e_{s}$ is saturation vapor pressure, and $\mathrm{e}_{a}$ is actual vapor pressure (Rosenberg et al. 1983). Temperatures and vpd for the wrapper and midframe leaves were estimated from measurements taken in the two adjacent strata by linear interpolation. Movements were observed over a wide range of environmental conditions during the $71 \mathrm{~d}$, although occasional equipment malfunctions prevented us from obtaining microclimate measurements for all of the movement data.

The objective was to estimate transition probabilities (i.e., the probability of moving from one stratum to the same or another stratum in $1 \mathrm{~d}$ ) as 
a function of these environmental conditions. We modeled the individual transition probabilities as definite integrals of the Beta probability density function (pdf), the shape parameters of which are a function of the stratum from which the larvae are moving and the environmental conditions. Assuming that all of the observed movements were independent events leads to the following likelihood function for all observed movements:

$$
\prod_{i} \prod_{t} \prod_{k} p_{j i k}^{\text {pijk }}
$$

where $p_{i j k}$ is the probability of moving from stratum $i$ to stratum $j$ on day $k$, and $n_{i j k}$ is the number of larvae observed to have moved from stratum $i$ to stratum $j$ on day $k$. The stratum indicators, $i$ and $j$, take integer values from 1 to 5 corresponding to the head, wrapper leaves, upper frame, midframe, and lower frame leaves respectively. The individual transition probabilities are modeled as:

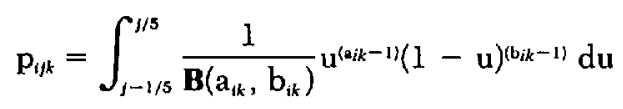

a definite integral of the Beta pdf with parameters $a_{i k}$ and $b_{i k}$ (here $\mathbf{B}(a, b)$ represents the beta function). The Beta pdf was chosen for its range (0 to 1) which allows convenient limits of integration (a simple function of $j$ ) and for its very flexible shape. Probabilities of moving from any stratum $i$ to any stratum $j$ may be high in some conditions; whereas in other conditions, the same probability may be low. The shapes that the Beta pdf can assume are varied enough for these different cases to be possible under the model described above. These shapes are not unlimited, however, so some structure is forced on the probabilities (we return to this point in the ensuing discussion). We hypothesize that movements of the larvae depend on environmental conditions, suggesting that the shape parameters for the Beta pdf should change with changing conditions. The shape parameters are modeled as functions of environmental conditions according to:

$$
\begin{aligned}
& \mathrm{a}_{i k}=\exp \left(\mathbf{c}^{\prime}{ }_{i} \mathbf{x}_{i k}\right) \\
& \mathbf{b}_{i k}=\exp \left(\mathbf{d}^{\prime}{ }_{i} \mathbf{x}_{i k}\right)
\end{aligned}
$$

where $\mathbf{x}_{i k}$ is a vector of measurements of environmental conditions and $c_{i}$ and $d_{i}$ are the corresponding parameter vectors that are to be estimated. The exponential function in Equations 4 and 5 was chosen for two reasons. First, it results in parameter estimates greater than zero, and the Beta shape parameters must be greater than or equal to zero. Second, by speculating about the shape changes in the Beta pdf that might be necessary to fit this model, we concluded that, for some strata, large changes in the values of parameters may be necessary over a narrow range of environmental conditions (e.g., little change in the shape parameters as temperature increases until it rises above a threshold, and then sudden large changes in shape parameters corresponding with large changes in movement patterns).

The likelihood function was obtained by substituting the right side of Equations 4 and 5 for $a_{i k}$ and $b_{i k}$, respectively, in Equation 3, using the measurements of microclimate conditions in the research plot canopy for the $x_{i k}$, then substituting the right side of Equations 3 for $p_{i j k}$ and the data on larval movements for the $\mathbf{n}_{. . k}$ in Equation 2. The total number of larval movements used in the analysis $\left(\sum_{k}\right)$ was 1,186 . The $\mathbf{c}_{i}$ and $\mathbf{d}_{\boldsymbol{i}}$ were then estmated by the method of maximum likelihood; a computer program (GAUSS, Aptech Systems, Inc., Kent, Wash.) was used to minimize minus the log of Equation 2 over the values of the elements in $\mathbf{c}_{i}$ and $\mathbf{d}_{1}$. Maximum likelihood estimation yields the most likely values for the parameters, given the form of the model chosen and the data collected. Several alternate forms if the $\mathbf{x}_{i k}$ vector were considered, including:

$$
\begin{aligned}
\mathbf{x}_{i k} & =\left(\text { constant }_{i}, \text { temp }_{i k}, \operatorname{vpd}_{i k}\right) \\
& =\left(\mathbf{x}_{i k 1}, \mathbf{x}_{i k 2}, \mathbf{x}_{i k 3}\right) \\
\mathbf{x}_{i k} & =\left(\text { constant }_{i}, \text { temp }_{i k}\right) \\
& =\left(\mathbf{x}_{i k 1}, \mathbf{x}_{i k 2}\right) \\
\mathbf{x}_{i k} & =\left(\text { constant }_{i}, \mathrm{vpd}_{i k}\right) \\
& =\left(\mathbf{x}_{i k 1}, \mathrm{x}_{i k 3}\right) \\
\mathbf{x}_{i k} & =(\text { constant } \\
& =\left(\mathbf{x}_{i k 1}\right)
\end{aligned}
$$

where temp $p_{i k}$ is the standardized average temperature estimated for stratum $i$ on day $k$; and $\mathrm{vpd}_{i k}$ is the standardized average vpd estimated for stratum $i$ on day $k$. Average temperature and $v p d$ were standardized by subtracting the mean and dividing by the standard deviation of all daily averages measured, to condition better the likelihood function for parameter estimation.

We compared the full and reduced models given by Equation 6 through 9 using generalized likelihood ratio tests. If environmental conditions do not correlate well with transition probabilities, we expect these tests to show that the full models (given by Equations 6 through 8 ) are not necessary; hence, the most reduced model (that including a constant and no measurements of environmental conditions) would be sufficient.

To assess the ability of the model to predict frequency of movement by $T$. $n i$ larvae, we evaluated standardized cell residuals given by

$$
\frac{\begin{array}{c}
\text { (observed no. movements } \\
\text { predicted no. movements) }
\end{array}}{\sqrt{\text { predicted }}}
$$

as described by Bishop et al. (1975). These residuals approximate a standard normal distribution. If the approximation is adequate, most of the residuals should be within the range of -2 to because most of the standard normal distribution falls within this 
range, so the range of -2 to 2 provides the criterion for evaluating fit of the model. Residuals were generated from the observations used in parameter estimation and predictions of these observations given by the model. Environmental conditions for these predictions were given by hourly temperature measurements taken in the crop canopy, and for comparison, by the daily average temperature measurements taken at a weather station about 3 $\mathrm{km}$ from the research plots.

An important question when constructing a model that will be used for prediction is how well it will work in conditions different from those under which the model was parameterized. To address this, we observed movement of late instars of $T$. $n i$ on the spring cabbage crop at the University of Florida's Gulf Coast Agricultural Research and Education Center in Bradenton, Fla. The differences between these conditions and those encountered in Geneva, N.Y., were as follows: the plants were at an earlier growth stage, with the heads just beginning to form (stages 6-7, Andaloro et al. 1983); the average temperatures in these plots were just above the upper limits of the temperatures encountered in Geneva; and the cabbage in Bradenton was grown in sandy soil which was ridged and covered with black plastic mulch (as opposed to uncovered clay-loam soil with very little ridging in Geneva). Larvae used in these experiments were from the same culture as those used in the Geneva experiments and were released in the plots in the first instar. On any given plant, approximately 10 larvae were released on the same stratum, and an equal number of releases were made on each of the strata by releasing larvae on different plants. The larvae were not labeled in any way, and no attempt was made to confine the larvae on any one stratum until they reached the late instars. The release strategy in this case was designed to result in different intraplant distributions of larvae on the different plants at the start of the experiment.

In these plots, we observed net movement by counting the number of larvae on each of the five vertical strata on 20-30 plants in the late afternoon of one day, then repeating the sample on the same plants $24 \mathrm{~h}$ later. At the same time, temperature within the crop canopy was measured by the same equipment and methods used in the Geneva plots. In this case, the probability of a given larva being found on stratum $j$, plant $r$, is

$$
\begin{aligned}
& \frac{\mathrm{n}_{\mathrm{lr}}}{\mathrm{n}_{r}} \times \mathrm{p}_{1 j}+\frac{\mathrm{n}_{2 r}}{\mathrm{n}_{r r}} \\
& \quad \times \mathrm{p}_{2 j}+\ldots+\frac{\mathrm{n}_{5 r}}{\mathrm{n}_{r}} \times \mathrm{p}_{5 j}
\end{aligned}
$$

where $\mathrm{n}_{\mathrm{ir}}$ is the number of larvae on stratum $i$ and plant $r$ in the first sample, $n_{r}$ is the total number of larvae on plant $r$ in the first sample, the $\frac{n_{i r}}{n_{r}}$ are the probabilitifs of finding a given larva on stratum $i$ and plant $r$ in the first sample, and $p_{i j}$ is the probability of a larva from stratum $i$ in the first sample being found on stratum $j$ during the second sample. This leads to the following likelihood function:

$$
\prod_{j} \prod_{r}\left(\sum_{i} \frac{n_{t r}}{n_{. r}} \times p_{i j}\right)^{m_{j r}}
$$

where $\mathrm{n}_{i r}$ is the number of larvae on plant $r$ and stratum $i$ on day $1, \mathrm{n}_{r}$ is the total number of larvae on plant $r$ on day 1 , and $m_{i r}$ is the number of larvae on plant $r$ and stratum $j 24 \mathrm{~h}$ later. As in Equation 3 , the $\mathrm{p}_{i j}$ are modeled as definite integrals of the Beta pdf but with exponentiated shape parameters to force the values of these parameters to be greater than zero,

$$
\begin{aligned}
p_{i j}= & \int_{J-1 / 5}^{j / 5} \frac{1}{B\left(\exp \left(\mathrm{a}_{i}^{*}\right), \exp \left(\mathrm{b}_{i}^{*}\right)\right.} \mathrm{u}^{\left(\exp \left(*^{*}\right)-1\right)} \\
& \cdot(1-\mathrm{u})^{\left(\exp \left(\mathrm{b}^{*}\right)-1\right)} \mathrm{du}
\end{aligned}
$$

where $a^{*}$, and $b^{*}$, are the parameters estimated by maximizing Equation 12 over their values. The total number of larvae observed $\left(\sum n_{r}\right)$ for each estimation ranged from 107 to 246 . Note that we are only estimating shape parameters for a single day with Equations 12 and 13. These shape parameters were compared with those predicted for the temperature conditions in Bradenton by the model parameterized in Geneva.

\section{Results and Discussion}

Values estimated for the $c_{i}$ and $d_{i}$, the coefficients for environmental variables as described in Equations 4 and 5 , are given in Table 1. Estimated coefficients for temperature and vpd were not zero, and likelihood ratio tests showed that these measurements of microclimate conditions significantly increased the values of the likelihood functions, indicating that they should be included in the model. Likelihood ratio tests also showed that, for most strata, the models containing a constant and the combination of temperature and vpd had significantly greater maximized likelihoods than models containing either vpd or temperature and a constant. While nonnested models cannot be compared by the likelihood ratio test, the maximized likelihood for models containing vpd and a constant were usually considerably higher than those for models containing temperature and a constant. For one stratum (the wrapper leaves), the model with temperature alone had a higher value of the maximized likelihood than the model containing vpd alone, but for two other strata (the head and upper frame leaves), the increase in the value of the likelihood function for the model including temperature and a constant over that for the model containing only a constant was not significant $(P>$ 0.25 ). However, if this model were used for predicting frequency of larval movement, consideration would have to be given to the relative diffi- 
Table 1. Maximized values of likelihood functions and associated coefficients of environmental measurements used to calculate shape parameters for the Beta pdf in a model of transition probabilities for $T$. ni larvae on cabbage plants

\begin{tabular}{|c|c|c|c|c|c|c|c|}
\hline \multirow{3}{*}{ Stratum } & \multicolumn{6}{|c|}{ Coefficients from Equations 4 and 5 for } & \multirow{3}{*}{$\begin{array}{c}\text {-Log } \\
\text { likelihood }\end{array}$} \\
\hline & \multicolumn{3}{|c|}{ Parameters $\left(c_{1}\right)$ for $a_{1 k}$} & \multicolumn{3}{|c|}{ Parameters $\left(\mathrm{d}_{i}\right)$ for $\mathrm{b}_{i k}$} & \\
\hline & Constant & Temp & $\begin{array}{c}\text { Vapor } \\
\text { pressure } \\
\text { deficit }\end{array}$ & Constant & Temp & $\begin{array}{c}\text { Vapor } \\
\text { pressure } \\
\text { deficit }\end{array}$ & \\
\hline Head & $\begin{array}{l}0.217 \\
0.184 \\
0.253 \\
0.221\end{array}$ & $\begin{array}{l}0.167 \\
0.080 \\
- \\
-\end{array}$ & $\begin{array}{c}-0.889 \\
-0.809 \\
-\end{array}$ & $\begin{array}{l}1.560 \\
1.534 \\
1.647 \\
1.645\end{array}$ & $\begin{array}{r}0.086 \\
-0.012 \\
- \\
-\end{array}$ & $\begin{array}{c}-1.003 \\
- \\
-0.098 \\
-\end{array}$ & $\begin{array}{l}132.86^{*} \\
136.74 \mathrm{~ns} \\
133.46^{* *} \\
137.31\end{array}$ \\
\hline $\begin{array}{c}\text { Wrapper } \\
\text { leaves }\end{array}$ & $\begin{array}{l}1.746 \\
1.751 \\
1.673 \\
1.697\end{array}$ & $\begin{array}{l}0.270 \\
0.141 \\
- \\
-\end{array}$ & $\begin{array}{l}0.075 \\
- \\
0.153 \\
-\end{array}$ & $\begin{array}{l}2.574 \\
2.550 \\
2.501 \\
2.503\end{array}$ & $\begin{array}{l}0.058 \\
0.052 \\
- \\
-\end{array}$ & $\begin{array}{c}-0.016 \\
- \\
0.067 \\
-\end{array}$ & $\begin{array}{l}304.39^{* *} \\
310.04^{* *} \\
320.07^{* *} \\
326.18\end{array}$ \\
\hline $\begin{array}{l}\text { Upper frame } \\
\text { leaves }\end{array}$ & $\begin{array}{l}1.715 \\
1.886 \\
1.800 \\
1.882\end{array}$ & $\begin{array}{c}-0.188 \\
-0.000 \\
- \\
-\end{array}$ & $\begin{array}{l}0.404 \\
- \\
0.268 \\
-\end{array}$ & $\begin{array}{l}1.699 \\
1.878 \\
1.790 \\
1.879\end{array}$ & $\begin{array}{c}-0.234 \\
-0.041 \\
- \\
-\end{array}$ & $\begin{array}{l}0.414 \\
- \\
0.267 \\
-\end{array}$ & $\begin{array}{l}258.40^{* *} \\
269.00 \mathrm{~ns} \\
267.20^{*} \\
269.52\end{array}$ \\
\hline $\begin{array}{l}\text { Middle frame } \\
\text { leaves }\end{array}$ & $\begin{array}{l}2.215 \\
2.545 \\
2.389 \\
2.556\end{array}$ & $\begin{array}{c}-0.472 \\
-0.541 \\
- \\
-\end{array}$ & $\begin{array}{l}0.470 \\
- \\
0.260 \\
-\end{array}$ & $\begin{array}{l}1.270 \\
1.681 \\
1.428 \\
1.685\end{array}$ & $\begin{array}{c}-0.476 \\
-0.535 \\
- \\
-\end{array}$ & $\begin{array}{l}0.627 \\
- \\
0.430 \\
-\end{array}$ & $\begin{array}{l}160.85^{* *} \\
183.22^{* * *} \\
172.98^{* * *} \\
200.07\end{array}$ \\
\hline $\begin{array}{l}\text { Lower frame } \\
\text { leaves }\end{array}$ & $\begin{array}{l}1.277 \\
1.326 \\
1.101 \\
1.333\end{array}$ & $\begin{array}{l}0.893 \\
1.025 \\
- \\
-\end{array}$ & $\begin{array}{l}0.418 \\
- \\
0.326 \\
-\end{array}$ & $\begin{array}{l}-1.182 \\
-1.080 \\
-1.507 \\
-1.166\end{array}$ & $\begin{array}{l}1.263 \\
1.439 \\
- \\
-\end{array}$ & $\begin{array}{l}0.724 \\
- \\
0.637 \\
-\end{array}$ & $\begin{array}{l}83.54^{* *} \\
89.48^{*} \\
85.65^{* *} \\
92.35\end{array}$ \\
\hline
\end{tabular}

a The null hypothesis that the coefficient(s) for temperature or vapor pressure deficit or both are zero can be rejected at: ${ }^{*}, P \leq$ $0.01 ; *, P \leq 0.05 ;$ ns, cannot reject at $P \leq 0.05$.

culty of measuring or predicting vpd as compared with temperature in selecting between the candidate models. Because temperature predictions are so much more readily available than predictions of vpd, we evaluated the predictive ability of the model using temperature alone.

Most of the standardized cell residuals were between -2 and 2 for predictions based on temperatures measured in research plots as well as those based on temperature measurements from a nearby weather data recording station. Occasional cases where the magnitude of deviations was greater than 2 occurred when the predicted probability of movement was very small, or when the total observed number of larvae on a given stratum was small, resulting in a very small (often fractional) number of larvae predicted to move. When the number of larvae predicted to move was a small fraction, and when only one larva was observed to move, the resulting deviation was large. However, Bishop et al. (1975) warn that these cases are the most difficult to interpret according to the asymptotic theory behind the technique. The important result is that the model predicts the data well. If we were able to include good predictions of vpd, the fit would improve.

Fig. 1 gives the estimated relationship of the Beta pdf shape parameters to temperature, and Fig. 2 shows examples of the Beta pdf's predicted for two different temperatures. The exponential functions (Equations 4 and 5) used to model the Beta pdf shape parameters as functions of microclimate were quite flexible, giving relationships of the parame- ters to temperature that ranged from almost linear to very nonlinear (Fig. 1). Fig. 2 demonstrates the flexibility in shapes that the Beta pdf can assume for different strata and different environmental conditions, making it a useful modeling tool in this analysis. The effects of these different shapes in the distributions in Fig. 2 on predicted transition probabilities are evident in the different areas under the curves between 0 and $0.2,0.2$ and 0.4 , etc.

The results provide useful insight into the spatial dynamics of $T, n i$ larvae on cabbage plants. The transition probabilities for high temperatures and vapor pressure deficits indicate that movement tends to be downward toward the relatively shaded and cool environment in the lower frame leaves; at low temperatures and vapor pressure deficits, movement tends to be upward toward the relatively sunny and warm environment at the top of the plant (Fig. 2). These behaviors would result in maintenance of a more narrow range of body temperature and a water balance; in turn, the likelihood of feeding damage on the marketable parts of the plant seem to be affected by these behaviors.

The model used in this analysis represents a new method of estimating transition probabilities as a function of other variables. It forces a structure, imposed by the shapes the Beta pdf can assume, on the transition probabilities. This structure is quite useful for the data we collected because it was present in the data: The highest proportion of larvae did not move, the next highest proportion moved one stratum, and the proportion of larvae moving two or more strata was almost always quite 
small. An alternative method would have been to estimate each transition probability individually as a function of environmental conditions using logistic regression. To estimate an entire $n \times n$ (in our case $5 \times 5$ ) matrix using logistic regression would require at least $2 n^{2}-2 n$ parameters (in our case 40 , estimate a constant and linear coefficient of one measure of environmental conditions for the first four probabilities in each column, and obtain the last element in each column by subtraction because the column must sum to one). Using the model described here, we obtained the entire matrix estimating only $4 n$ (in our case 20 ) parameters. The advantage should be more precision in estimating each parameter because each is estimated with more data. This advantage becomes particularly important if we increase spatial resolution or scale. For example, if we require a $10 \times 10$ matrix, we must estimate at least 180 parameters to obtain the entire matrix as a function of environmental conditions using logistic regression. Using the model described herein, we would have to estimate only 40 .

In Fig. 3, the Beta pdf's predicted by the model parameterized in Geneva are compared with Beta pdf's estimated from data collected in Bradenton. The probabilities of movement appear to be different for the two locations at $23^{\circ} \mathrm{C}$; larvae in Bradenton had a greater probability of moving upward from the lower strata on the plant. The conditions under which the Florida cabbage was grown could explain this difference in behavior between Bradenton and Geneva. The black plastic at the base of the plants in Bradenton absorbs more heat than moist bare soil, typical of the plots in Geneva. This results in a different microclimate in the canopy in Bradenton than in Geneva; i.e., higher temperatures and probably lower vpd at the base of the plant. The response of larvae in Geneva was to move away from high temperatures and low vpd, and the larvae in Bradenton seem to respond the same way to the higher temperatures near the black plastic mulch. Because the model assumes a certain consistent relationship of temperature in one part of the canopy to each of the other parts, the predictions made on the basis of that relationship in Geneva are not valid in Bradenton, where the relationship is different. These results suggest that the model would have to be reparameterized wherever the canopy microclimate profile is different. Canopy microclimate profile, however, may remain similar throughout a region; e.g., the canopy profile in Geneva should be typical for most of the northern United States and Canada. Other conditions that interact with temperature and vpd in the crop canopy (e.g., wind, solar radiation), and possibly differences in behavior of $T$. $n i$ biotypes, also could affect transition probabilities and the parameter estimates obtained for the model.

This model can be used for predicting frequency of insect movement as a function of predicted environmental conditions in a simulation model. If

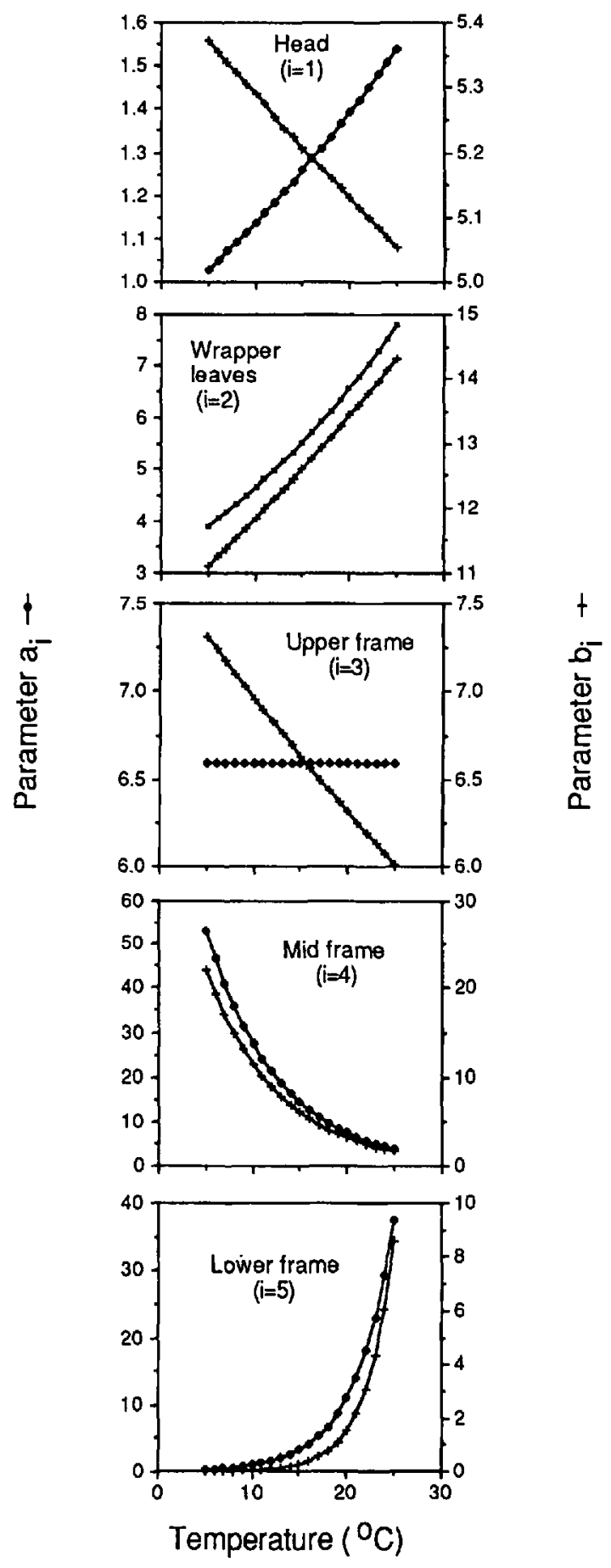

Fig. 1. Shape parameters for Beta probability den. sity functions used to model (see text) transition probabilities for $T$. ni on cabbage plants as a function of microclimate.

combined with submodels of development and foliage consumption, we can predict if and when feeding damage will occur on the marketable parts of the cabbage plant. Currently, we are constructing and validating this simulation model for use in 

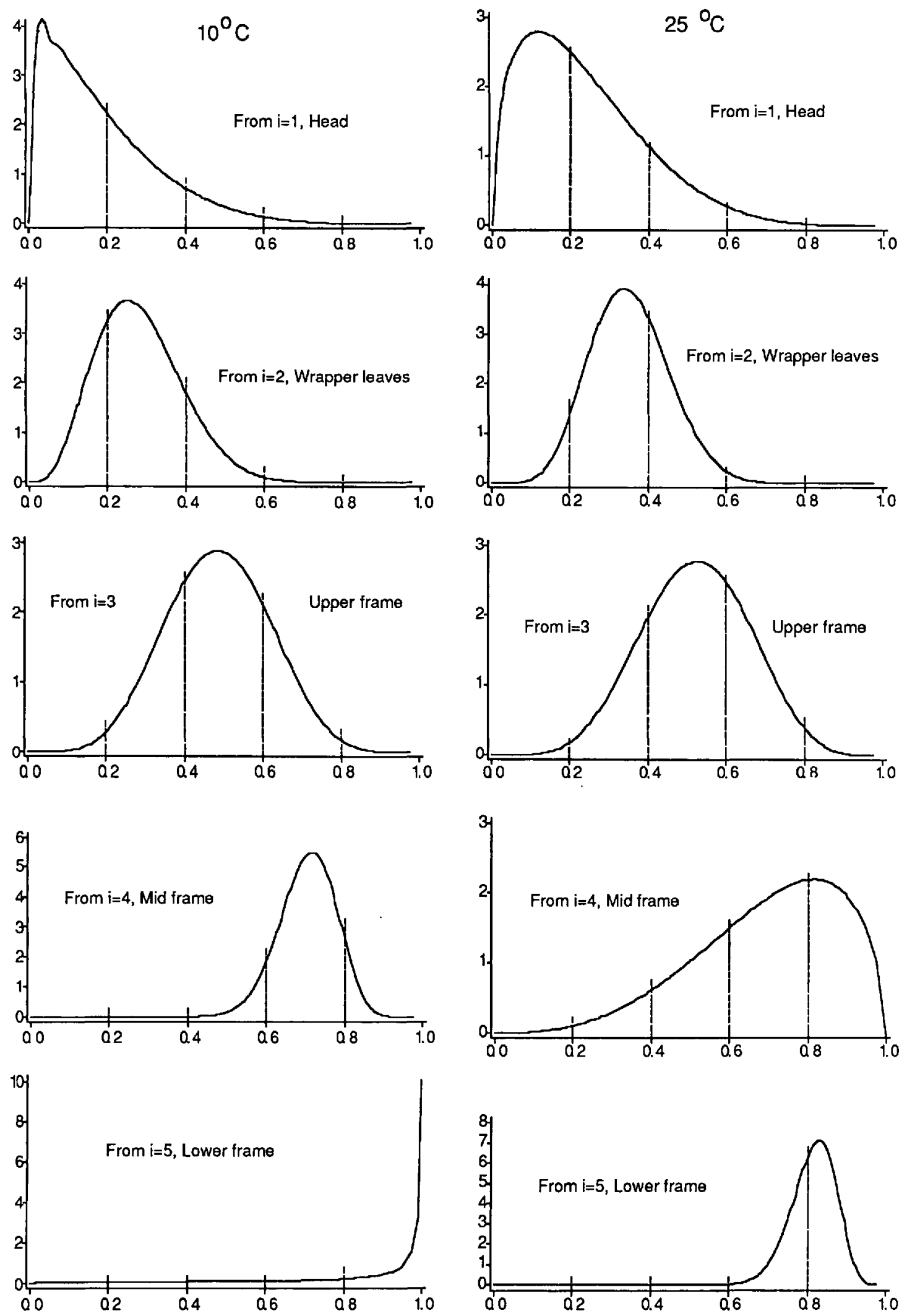

Fig. 2. Estimated Beta probability density functions at two different temperatures used to model transition probabilities for $T$. $n i$ on cabbage. Transition probabilities from the strata indicated on each plot to each of the other strata are obtained by integrating the area under the curve over the following limits: to the head, $0-0.2$; to the wrapper leaves, $0.2-0.4$; to the upper frame, $0.4-0.6$; to the midframe, $0.6-0.8$; to the lower frame, $0.8-1.0$. 

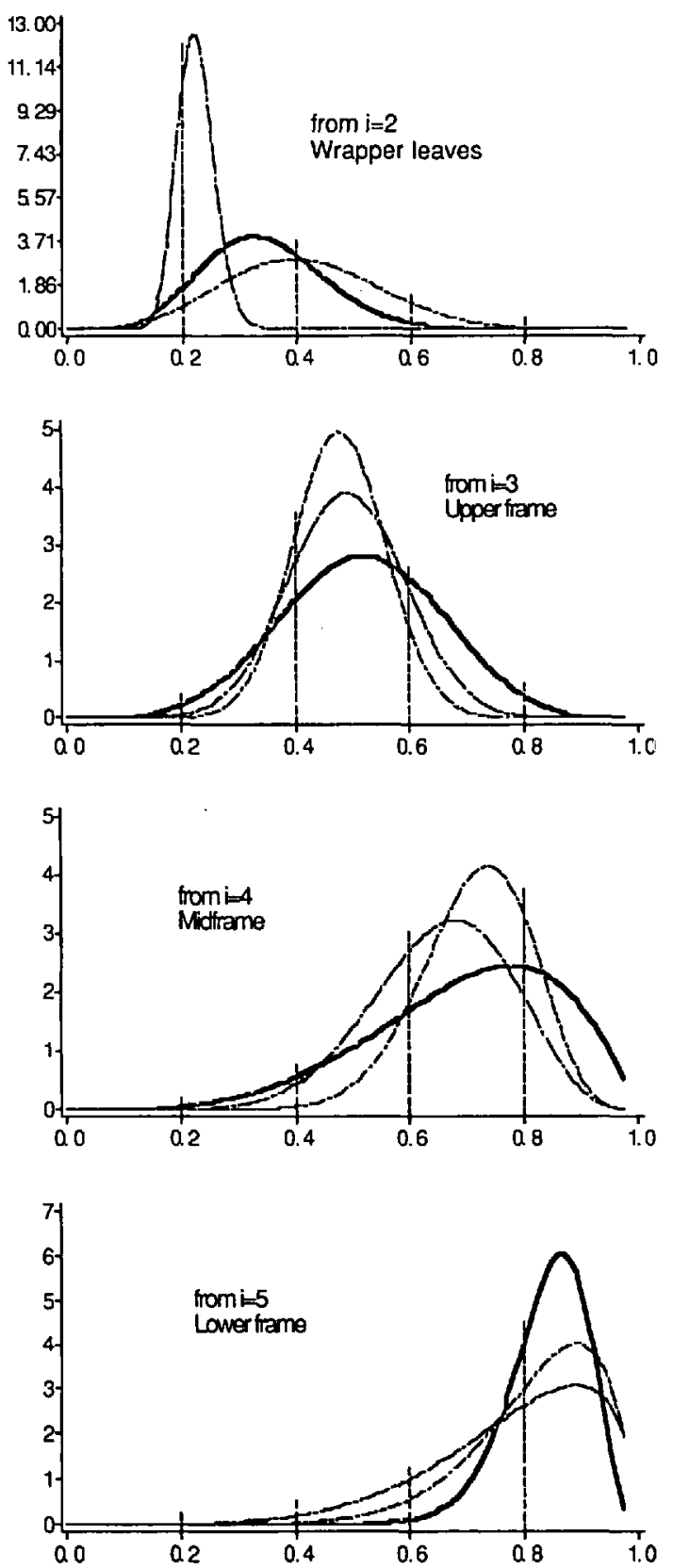

Fig. 3. Comparison of Beta probability density function predicted from a model parameterized in Geneva, N.Y., for an average temperature of $23^{\circ} \mathrm{C}$ (solid line) with Beta probability density functions estimated from data collected on two different days in Bradenton, Fla., at an average temperature of $23^{\circ} \mathrm{C}$ (dashed lines). On both days, so few larvae were found on the head that transition probabilities from this stratum could not be estimated.

cabbage pest management and to further our understanding of the spatial dynamics and population ecology of the larval stages of this species.

Understanding and predicting the spatial dynamics of insects is a difficult and important chal- lenge. The model described herein can help meet that challenge in a way that is mathematically sophisticated yet is intuitively appealing and graphically easy to understand. It is potentially useful in many other analyses concerning population spatial dynamics in biology and ecology.

\section{Acknowledgment}

We thank D. Knipple and D. Versage for their kind help with the radioisotope work; D. J. Schuster for graciously providing the research plots in Bradenton, Fla.; and A. J. Sawyer for helpful discussions. This work is part of a thesis submitted by C.W.H. to the Cornell University Graduate School in partial fulfillment of the requirements for the degree of Doctor of Philosophy.

\section{References Cited}

Alwood, W. B. 1886. Notes on insects and insecticides, pp. 214-215. In Ohio Agricultural Experiment Station Report 5.

Andaloro, J. T., K. B. Rose, A. M. Shelton, C. W. Hoy \& R. F. Becker. 1983. Cabbage growth stages. New York Food and Life Sciences Bulletin 101.

Anonymous. 1945. United States standards for cabbage. Source: $\$ \$ 51.450$ to 51.464 at 18 F. R. 7084 , Nov. 11, 1953.

Bishop, Y. M. M., S. E. Fienberg \& P. W. Holland. 1975. Discrete multivariate analysis. Massachusetts Institute of Technology Press, Cambridge.

Casey, T. M. 1976. Activity patterns, body temperature, and thermal ecology in two desert caterpillars. Ecology 57: 485-497.

Cloudsley-Thompson, J. L. 1962. Microclimates and the distribution of terrestrial arthropods. Annu. Rev. Entomol. 7: 199-222.

1975. Adaptations of Arthropoda to arid environments. Annu. Rev. Entomol. 20: 261-283.

Greene, G. L. 1968. Distribution of Trichoplusia $n i$ eggs and larvae on cabbage plants as a basis for sampling efficiency. J. Econ. Entomol. 61: 1648-1650.

Hayslip, N. C., W. G. Genung, E. G. Kelsheimer \& J. W. Wilson. 1953. Insects attacking cabbage and other crucifers in Florida. Florida Agricultural Experiment Station Bulletin 534.

Hoy, C. W. \& A. M. Shelton. 1987. Feeding response of Artogeia rapae (Lepidoptera: Pieridae) and Trichoplusia ni (Lepidoptera: Noctuidae) to cabbage leaf age. Environ. Entomol. 16: 680-682.

May, M. L. 1979. Insect thermoregulation. Annu. Rev. Entomol. 24: 313-349.

Reid, W. J., Jr., \& F. P. Cuthbert, Jr. 1957. Control of caterpillars on commercial cabbage and other cole crops in the south. USDA Farmer's Bulletin. 2099.

Rosenberg, N. J., B. L. Blad \& S. B. Verma. 1983. Microclimate the biological environment. Wiley, New York.

Sirrine, F. A. 1894. Insects affecting late cabbage, pp. 667-671. In New York State Agricultural Experiment Station Bulletin 83.

Sutherland, D. W. S. 1965. Biological investigations of Trichoplusia ni (Hübner) and related and associated species damaging cruciferous crops on Long Island, NY 1960-1963. Ph.D. dissertation, Cornell University, Ithaca, N.Y.

Received for publication 29 February 1988; accepted 27 June 1988. 